\title{
Editorial
}

\section{The Role of Surgery in Osteoarthritis, Rheumatoid Arthritis and Ankylosing Spondylitis}

\author{
W.S. Khan ${ }^{*}, 1$, and U.G. Longo ${ }^{2}$ \\ ${ }^{1}$ University College London Institute of Orthopaedics and Musculoskeletal Sciences, Royal National Orthopaedic \\ Hospital, Stanmore, Middlesex, HA7 4LP, UK \\ ${ }^{2}$ Department of Orthopaedic and Trauma Surgery, Campus Bio-Medico University, Via Alvaro del Portillo, 200, 00128 \\ Trigoria, Rome, Italy
}

Surgery has an important role in the management of different forms of arthritis including osteoarthritis, rheumatoid arthritis, and sero-negative and crystal arthropathies.

The role of surgery in the management of arthritis begins with prevention, and in the case of osteoarthritis it is important to ensure fractures and injuries are managed appropriately to prevent post-traumatic degenerative changes in joint [1-3]. Pedersen et al. discuss acute osteochondral fractures in the lower extremities and provide an approach to the identification and treatment of these injuries. Chondral and osteochondral fractures of the lower extremities are important injuries because they can cause pain and dysfunction and often lead to osteoarthritis. These injuries can be misdiagnosed initially which may impact on the healing potential and result in poor long-term outcome. Their comprehensive review focuses on current pitfalls in diagnosing acute osteochondral lesions, potential investigative techniques to minimize diagnostic errors as well as surgical treatment options. Acute osteochondral fractures are frequently missed and can be identified more accurately with specific imaging techniques. A number of different methods can be used to fix these fractures but attention to early diagnosis is required to limit progression to osteoarthritis. These fractures are common with joint injuries and early diagnosis and treatment should lead to improved long term outcomes.

Another important facet of prevention is the prevention of fractures by appropriate treatment of bone lesions [4]. Management of unicameral bone cysts remains controversial. These cysts seldom heal spontaneously or even after pathological fracture. Sometimes these cysts can be very large and incredibly troublesome to the patient. Various treatments exist with variable success rates. Zaghloul et al. describe a novel minimally invasive technique for the management of these lesions. The authors present their

\footnotetext{
*Address correspondence to this author at the University College London Institute of Orthopaedics and Musculoskeletal Sciences, Royal National Orthopaedic Hospital, Stanmore, Middlesex, HA7 4LP, UK;

Tel: 07791 025554; Fax: 01707 655059;

E-mail:wasimkhan@doctors.org.uk

${ }^{\S}$ Guest Editor
}

experience of treating these lesions by continuous drainage. Six patients with unicameral bone cysts were treated by inserting a modified drain into the wall of the cyst. The aim of surgery was to place the drain in a dependent area of the cyst, through the cortex allowing for continuous drainage. This was achieved through a small incision under radiographic control, a cement restrictor to prevent closure of the drain site, and a redivac drain. The drain was left in place for a week to establish an epithelialized pathway. Five cases were completely healed. Only one had a further fracture and there were no recurrent fractures. All the patients reported complete comfort and they all were able to re-engage in recreational activities without restriction. The authors conclude that reducing the intra-medullary pressure in these lesions will lead to healing, and report a safe and minimally invasive technique for the management of these lesions.

Once arthritis is established, there is a number of treatment options available including arthrodesis $[2,5]$. First metatarsalphalangeal joint arthrodesis is a well-established and successful treatment; however there still remains controversy over the best choice of construct [6]. Rashid et al. describe a retrospective comparison of two methods of fixation. They performed a retrospective study of 52 patients undergoing first metatarsalphalangeal fusion using either dorsal non-locking plate with additional compression lag screw fixation or dorsal non-locking plate alone. They found when assessing clinical criteria, patients with dorsal nonlocking plates and additional compression lag screw fixation had a significantly higher rate of fusion, significantly higher rate of fusion within the first two months, significantly earlier time to fusion, and significantly lower rate of nonunion. When blindly assessing radiographic criteria, the patients treated with the plate and compression screw had a significantly higher rate of fusion and lower rate of nonunion. There was no statistically significant difference between the frequencies of complications in the groups. The authors conclude that the interfragmentary compression is a crucial factor in achieving good union rates and recommend the use of non-locking pre-contoured plating with additional interfragmentary compression screw as the fixation method of choice for these procedures.

Joint replacement is another option but can present different challenges in different arthropathies [7, 8]. Hip replacement surgery is increasingly performed with better 
results [9]. Putnis et al. present a literature review of total hip arthroplasty in patients with ankylosing spondylitis. Ankylosing spondylitis is a spondyloarthropathy affecting the sacro-iliac joints initially, with subsequent progression to the spine and the hip joints. The hip joints are affected by synovitis, enthesial inflammation, and involvement of medullary bone, progressive degeneration and secondary osteoarthritis. Clinical presentation is usually in the form of pain and stiffness progressing to disabling fixed flexion contractures and, in some instances, complete ankylosis. Hip arthroplasty could be considered for hip pain, postural and functional disability, or pain in adjacent joints due to hip stiffness. The authors conducted a literature review to determine peri-operative considerations and outcome in ankylosing spondylitis patients undergoing hip arthroplasty. They discuss peri-operative considerations including preoperative surgical planning, thromboprophylaxis, anaesthetic considerations and heterotopic ossification. They also discuss outcomes including range of movement, pain relief, survivorship and complications.

There have been many developments in the management of knee pathologies [10-12] and knee replacements are increasingly being performed for end stage knee arthritis [13]. Donaldson et al. discuss current controversies of alignment in total knee replacements. In the majority of patients, knee replacement alleviates pain and improves function. However up to $20 \%$ of patients remain dissatisfied, even with well-aligned and secure implants. Restoration of a neutral mechanical axis has traditionally been strived for, to improve both function and implant survival, and there is historical data to support this. More recently this view has been questioned and some surgeons are trying to improve function and outcomes by moving away from standard orthogonal alignment in an attempt to reproduce the kinematics of the pre-arthritic knee of that individual. Others are using computers, robots and patient specific guides to improve accuracy $[14,15]$. This article reviews the traditional alignment concept and the newer techniques, along with the evidence behind it.

An important consideration with any joint replacement is to avoid infection $[9,10]$. Since the introduction of laminar air flow in orthopaedic theatres by Sir John Charnley, it has widely become accepted as the standard during orthopaedic procedures such as joint arthroplasty. James et al. discussed the current evidence for the use of laminar flow in reducing infection rates in joint arthroplasty. The authors present a review of available current literature for the use of laminar flow operating theatre ventilation during total joint arthroplasty and examine the effectiveness of laminar flow ventilated operating theatres in preventing post-operative wound infection. Results of their findings suggest that while bacterial and air particulate is reduced by laminar air flow systems, there is no conclusive effect on the reduction of post-operative wound infections following total joint arthroplasty. They conclude that a combination of strict aseptic technique, prophylactic antibiotics and good anaesthetic control during surgery remains crucial to reduce post-operative surgical infections.

There have been a number of advances in joint replacements and surgeons are continuing to strive and identify how the outcomes could be improved [13]. Bilateral total knee arthroplasty can be performed either as a staged or simultaneous procedure. Haddad et al. describe a patientmatched retrospective observational study looking at bilateral simultaneous total knee arthroplasty. They compared the need for transfusion, the length of procedure, the length of stay, and complications of bilateral simultaneous knee arthroplasty with those of unilateral knee arthroplasty. Sixty-nine patients who underwent bilateral simultaneous knee arthroplasty procedures were compared with a matched control group of 69 patients who underwent unilateral knee arthroplasty. Receiver Operating Characteristic (ROC) curve was used to determine optimum cut-off values. Cumulative transfusion episodes were lower in the bilateral group than twice that of the unilateral group. In multivariate analysis the preoperative haemoglobin level and bilateral procedures were independent factors predicting the need for transfusion. The average length of procedure and length of hospital stay in the bilateral group was less than twice that of the unilateral group. Advanced age and bilateral procedures were independent predictors of prolonged length of stay. A haemoglobin level of $12.5 \mathrm{~g} / \mathrm{dL}$ and age of 70 were most suitable cut-off points to predict need for transfusion and occurrence of medical complications respectively. They concluded that bilateral simultaneous knee arthroplasties are safe and cost effective in appropriately selected patients. They recommend avoiding bilateral simultaneous procedures in patients over the age of 70 years, and with significant comorbidities.

As total knee arthroplasty is becoming an increasingly common procedure, revision surgery, particularly for infection, is associated with increasing morbidity and healthcare costs $[13,14]$. The current gold standard is a two stage revision procedure but single stage revision is increasingly being used in some departments to improve patient outcomes. Chew et al. conducted a systematic review of the literature to determine the up-to-date evidence underlying the use of a single stage knee approach in revision surgery. A total of 12 studies were included in this review amounting to 433 revision surgeries. This is the largest review of single stage knee revision surgery. The procedures described were heterogenous and included the 'two-in-one' technique as well as other single stage revision procedures. There were also differences in implants and antibiotic regimens. The mean re-infection rates described in 10 studies was $9.4 \%$ (range $0-19.2 \%$ ) after a mean follow-up of 40.3 months (range 7-180 months). The re-infection rates in the studies published over the last 30 years are falling, and this is not accounted for by any significant change in duration of follow-up over this period. The outcome scores used varied, but patients generally showed an improvement. The Knee Society Score and the Oxford Knee Score were the most commonly used in five and three studies respectively. We conclude that the current evidence for single stage revision is variable and there is a lack of good quality evidence to address whether single stage revisions is thorough enough to eradicate deep infection and able to restore adequate function. There is a need for larger prospective studies with standardised procedures and protocol, and with adequate follow-up. Till then, patients considered for a single stage approach should be thoroughly assessed and the surgery should be performed by a senior surgeon with experience in single stage knee revisions. 


\section{REFERENCES}

[1] Al-Rashid M, Khan WS, Vermulapali K. Principles of fracture fixation in orthopaedic trauma surgery. J Perioper Pract 2010; 20(3): 113-7.

[2] Khan WS, Oragui E, Akagha E. Common fractures and injuries of the ankle and foot: functional anatomy, imaging, classification and management. J Perioper Pract 2010; 20(7): 249-58.

[3] Martinelli N, Bonifacini C, Longo UG, et al. Current strategies of tissue engineering in talus chondral defects. Curr Stem Cell Res Ther 2013; 8(3): 217-21.

[4] Bhamra J, Malik AA, Khan WS, Pollock R. The perioperative management of skeletal metastases. J Periop Pract 2012; 22(1): 249 .

[5] Bhamra J, Bhamra K, Hindocha S, Khan WS. The role of wrist fusion and wrist arthroplasty in rheumatoid arthritis. Curr Rheumatol Rev 2015 (in press).

[6] Nash WJ, Al-Nammari S, Khan WS, Pengas IP. Surgical management of the forefoot in patients with rheumatoid arthritis - a review article. Open Orthop J 2015; 9: 84-9.

[7] Kennedy JW, Khan WS. Total hip arthroplasty in systemic lupus erythematosus: a systematic review. Int J Rheumatol 2015 (in press).
[8] Mosleh-Shirazi MS, Ibrahim M, Pastides P, Khan WS, Rahman H. An insight into methods and practices in hip arthroplasty in patients with rheumatoid arthritis. Int J Rheumatol 2015 (in press).

[9] Dheerendra S, Khan WS, Saeed MZ, Goddard NJ. Recent developments in total hip replacements: cementation, articulation, minimal-invasion and navigation. J Perioper Pract 2010; 20(4): 133-8.

[10] Malik A, Khan W. Editorial: recent advances and developments in knee surgery. Open Orthop J 2012; 6(2): 266-7.

[11] Onyema C, Oragui E, White J, Khan WS. Evidence-based practice in arthroscopic knee surgery. J Perioper Pract 2011; 21(4): 128-34.

[12] Jaiswal PK, Wong K, Khan WS. Current cell based strategies for knee cartilage injuries. J Stem Cells 2010; 5(4):177-85.

[13] Wong J, Khan WS, Chimutengwende-Gordon M, Dowd GSE. Recent advances in designs, approaches and materials in total knee replacement: Literature review and evidence today. J Perioper Pract 2011; 21(5): 165-71.

[14] Wong JM, Khan WS, Saksena J. The role of navigation in total knee replacement surgery. J Periop Pract 2013; 23(9): 202-7.

[15] Punwar S, Khan WS, Longo UG. The use of computer navigation in hip arthroplasty: literature review and evidence today. Orthop Traumatol Rehabil 2011; 13(5): 431-8.

(C) Khan et al.; Licensee Bentham Open.

This is an open access article licensed under the terms of the Creative Commons Attribution Non-Commercial License (http://creativecommons.org/licenses/by-nc/3.0/) which permits unrestricted, non-commercial use, distribution and reproduction in any medium, provided the work is properly cited. 